TRANSACTIONS OF THE

AMERICAN MATHEMATICAL SOCIETY

Volume 353, Number 8, Pages 3307-3325

S 0002-9947(01)02760-X

Article electronically published on April 10, 2001

\title{
BILINEAR ESTIMATES AND APPLICATIONS TO 2D NLS
}

\author{
J. E. COLLIANDER, J.-M. DELORT, C. E. KENIG, AND G. STAFFILANI
}

\begin{abstract}
The three bilinearities $u v, \overline{u v}, \bar{u} v$ for functions $u, v: \mathbb{R}^{2} \times[0, T] \longmapsto$ $\mathbb{C}$ are sharply estimated in function spaces $X_{s, b}$ associated to the Schrödinger operator $i \partial_{t}+\Delta$. These bilinear estimates imply local wellposedness results for Schrödinger equations with quadratic nonlinearity. Improved bounds on the growth of spatial Sobolev norms of finite energy global-in-time and blow-up solutions of the cubic nonlinear Schrödinger equation (and certain generalizations) are also obtained.
\end{abstract}

\section{INTRODUCTION}

The three bilinearities $u v, \overline{u v}, \bar{u} v$ for functions $u, v: \mathbb{R}^{2} \times[0, T] \longmapsto \mathbb{C}$ are studied in function spaces $X_{s, b}$ associated to the Schrödinger operator $i \partial_{t}+\Delta$. We establish sharp (up to endpoint) bilinear estimates for the $\mathbb{R}^{2}$ case extending the work of Kenig, Ponce and Vega [9] and Staffiliani [13]. These estimates imply local wellposedness of the initial value problems $(i=1,2,3)$

$$
\left\{\begin{array}{c}
i \partial_{t} u+\Delta u \pm N_{i}(u, \bar{u})=0 \\
u(0)=\phi \in H^{s}\left(\mathbb{R}^{2}\right)
\end{array}\right.
$$

with rough initial data $\phi$ where $N_{1}(u, \bar{u})=u^{2}, N_{2}(u, \bar{u})=\bar{u} u$ and $N_{3}(u, \bar{u})=\bar{u}^{2}$. The bilinear estimates also imply, following the arguments in 13], 12, polynomialin-time upper bounds on $\|u(t)\|_{H^{s}}, s \gg 1$, for certain global-in-time and blow-up solutions of the physical cubic nonlinear Schrödinger equation.

The proof of the bilinear estimates relies on a delicate geometric analysis. This analysis introduces new techniques which extend the calculus arguments first used in [8] and also in [9] to the $\mathbb{R}^{2}$ setting. In particular, we show how the support properties of the set where all the $X_{s, b}$ denominators simultaneously vanish may be exploited to prove sharp estimates. Similar ideas were used by Delort and Fang [7] for the Klein-Gordon equation. These techniques are also applied in a forthcoming paper on the Kadomtsev-Petviashvili I equation [6]. Related work which generalizes this study to higher dimensions and to other dispersive operators has recently been completed by T. Tao [14].

The next section contains the bilinear estimates and statements of the local wellposedness results concerning (1.1). Section 3 addresses regularity properties

Received by the editors July 24, 2000.

2000 Mathematics Subject Classification. Primary 35Q55, 42B35.

Key words and phrases. Nonlinear Schrödinger equation, nonlinear dispersive equations, weak turbulence, NLS blow-up, bilinear estimates, multilinear harmonic analysis, Strichartz inequalities.

J.E.C. was supported in part by an N.S.F. Postdoctoral Research Fellowship.

C.E.K. was supported in part by N.S.F. Grant DMS 9500725.

G.S. was supported in part by N.S.F. Grant DMS 9800879.

(C)2001 American Mathematical Society 
of solutions of the cubic nonlinear Schrödinger equation on $\mathbb{R}^{2}$. The third section also contains comments concerning higher power generalizations of cubic defocusing NLS stemming from the recent scattering result [11] of Nakanishi. We thank Kenji Nakanishi for a useful correspondence. We also thank Gustavo Ponce and Luis Vega for sharing their preliminary notes (in collaboration with C.E.K.) on the trilinear form $\overline{u v w}$ with $x \in \mathbb{R}$.

\section{Bilinear SchröDinger estimates on $\mathbb{R}^{2}$}

Let $u, v: \mathbb{R}^{2} \times[0, T] \longmapsto \mathbb{C}$. We consider here the three quadratic forms $u v, \overline{u v}, \bar{u} v$ and establish sharp bilinear estimates in the spaces $X_{s, b}$ associated to the Schrödinger operator $i \partial_{t}+\Delta$ on $\mathbb{R}^{2}$. These estimates extend the work of Kenig, Ponce and Vega [9] in one-dimension to the two-dimensional setting.

For $u: \mathbb{R}_{x}^{2} \times \mathbb{R}_{t}^{1} \longmapsto \mathbb{C}$, we recall the definition of the norm in $X_{s, b}$ from [1],

$$
\|u\|_{X_{s, b}}=\left(\iint\left|\left(1+\left.|\lambda+| k\right|^{2} \mid\right)^{b}(1+|k|)^{s} \widehat{u}(k, \lambda)\right|^{2} d k d \lambda\right)^{\frac{1}{2}}
$$

where $\widehat{u}$ denotes the space-time Fourier transform of $u$,

$$
\widehat{u}(k, \lambda)=\iint e^{-i(k x+\lambda t)} u(x, t) d x d t .
$$

Since $X_{s, b}$ is a weighted $L^{2}$ norm, we may replace all functions of the Fourier transform variables in the calculations of this norm by their absolute value. We will sometimes write $\alpha+$ to denote $\alpha+\epsilon$ and $\alpha$ - to denote $\alpha-\epsilon$ for arbitrarily small $\epsilon>0$.

This section establishes the following theorem.

Theorem 1. Let $b=\frac{1}{2}+$.

(i) The bilinear estimate

$$
\|\overline{u v}\|_{X_{\sigma, b-1}} \leq C\|u\|_{X_{s, b}}\|v\|_{X_{s, b}}
$$

holds provided $-\frac{1}{4}-(1-b)<s$, and $\sigma<\min \left(s+\frac{1}{2}+2(1-b), 2 s+2(1-b)\right)$. Moreover, the estimate (2.2) fails if $s<-\frac{1}{4}-(1-b)$ and also if

$$
\sigma>\min \left(s+\frac{1}{2}+2(1-b), 2 s+2(1-b)\right) \text {. }
$$

(ii) The bilinear estimate

$$
\|u v\|_{X_{\sigma, b-1}} \leq C\|u\|_{X_{s, b}}\|v\|_{X_{s, b}}
$$

holds provided $-\frac{1}{4}-(1-b)<s$, and $\sigma<\min \left(s+\frac{1}{2}, 2 s+2(1-b)\right)$. Moreover, the estimate (2.3) fails if $s<-\frac{1}{4}-(1-b)$ and also if $\sigma>\min \left(s+\frac{1}{2}, 2 s+2(1-b)\right)$.

(iii) The bilinear estimate

$$
\|\bar{u} v\|_{X_{\sigma, b-1}} \leq C\|u\|_{X_{s, b}}\|v\|_{X_{s, b}}
$$

holds provided $-\frac{1}{2}(1-b)<s$, and $\sigma<\min \left(s+\frac{1}{2}, 2 s+2(1-b)\right)$. Moreover, the estimate (2.4) fails if $s<-\frac{1}{2}(1-b)$ and also if $\sigma>\min \left(s+\frac{1}{2}, 2 s+2(1-b)\right)$.

In particular, we have the estimates

$$
\begin{aligned}
\|\overline{u v}\|_{X_{-\frac{1}{2}+,-\frac{1}{2}+}} & \lesssim\|u\|_{X_{-\frac{3}{4}+, \frac{1}{2}+}}\|v\|_{X_{-\frac{3}{4}+, \frac{1}{2}+}}, \\
\|u v\|_{X_{-\frac{1}{2}-,-\frac{1}{2}+}} & \lesssim\|u\|_{X_{-\frac{3}{4}+\frac{1}{2}+}}\|v\|_{X_{-\frac{3}{4}+\frac{1}{2}+}}
\end{aligned}
$$


and

$$
\|\bar{u} v\|_{X_{\frac{1}{4},-\frac{1}{2}+}} \lesssim\|u\|_{X_{-\frac{1}{4}+\frac{1}{2}+}}\|v\|_{X_{-\frac{1}{4}+\frac{1}{2}+}} .
$$

Using the technique used to prove Theorem 1.5 in [9] and also used in [8], the bilinear estimates of the theorem imply the following results concerning the local wellposedness of the initial value problems (1.1)

Corollary 2.1. In the cases $i=1,3$, the initial value problems (1.1) are locally wellposed for data in $H^{s}\left(\mathbb{R}^{2}\right), s>-\frac{3}{4}$. In case $i=2$, (1.1) is locally wellposed for data in $H^{s}, s>-\frac{1}{4}$.

Our proof of the estimates (2.2), (2.3) and (2.4) isolates common geometric features of all three estimates. In particular, a standard dyadic decomposition in the spatial frequency variable and a parabolic level set decomposition collapses consideration to four standard trilinear forms expressing the interactions of $L^{2}$ functions supported near paraboloids above dyadic shells. The main new difficulty in the $\mathbb{R}^{2}$ occurs when an orthogonal relationship among the vectors $k, k_{1}, k_{2}$ holds.

We outline the proof. We define the trilinear forms $A_{ \pm}, B_{ \pm}, C_{ \pm}$and then show that the estimates (2.2), (2.3) and (2.4) collapse to estimating $A_{+}, B_{-}$and $C_{+}$on functions supported above dyadic shells in the spatial frequency variable. Next, we state and prove lemmas which provide estimates on the trilinear forms $A_{+}, B_{-}$ and $C_{ \pm}$. (The proofs of these lemmas contain the hard work.) The lemmas are then used to prove the bilinear estimates of the theorem. Finally, we present some examples which demonstrate the necessity of some of the parameter restrictions in the theorem.

Notation. For nonzero $\mu_{i}, \mu_{j} \in \mathbb{R}^{2}$, let $\alpha_{i j}$ denote the angle between $\mu_{i}$ and $\mu_{j}$. For dyadic $M_{0}, M_{1}, M_{2}$ write $M_{*}=\min \left(M_{0}, M_{1}, M_{2}\right)$ and $M^{*}=\max \left(M_{0}, M_{1}, M_{2}\right)$. Subscripts on functions will sometimes be used to denote localizations, e.g., $f \chi_{\left\{\left|\mu_{0}\right| \sim M_{0}\right\}}=f_{M_{0}}$. Superscripts denote components of vectors. Finally, we write $\int_{*}$ to denote the expression

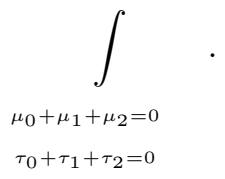

Standard Forms. The following expressions appear naturally in our study of bilinear Schrödinger estimates:

$$
\begin{aligned}
& A_{ \pm}(f ; g, h)=\int_{*} \frac{f\left(\mu_{0}, \tau_{0}\right)}{\left|\cos \alpha_{12}\right|^{1-b}} \frac{g\left(\mu_{1}, \tau_{1}\right)}{\left(1+\left.\left|\tau_{1}+\right| \mu_{1}\right|^{2} \mid\right)^{b}} \frac{h\left(\mu_{2}, \tau_{2}\right)}{\left(1+\left.\left|\tau_{2} \pm\right| \mu_{2}\right|^{2} \mid\right)^{b}}, \\
& B_{ \pm}(f ; g, h)=\int_{*} \frac{f\left(\mu_{0}, \tau_{0}\right)}{\left|\cos \alpha_{02}\right|^{1-b}} \frac{g\left(\mu_{1}, \tau_{1}\right)}{\left(1+\left.\left|\tau_{1}+\right| \mu_{1}\right|^{2} \mid\right)^{b}} \frac{h\left(\mu_{2}, \tau_{2}\right)}{\left(1+\left.\left|\tau_{2} \pm\right| \mu_{2}\right|^{2} \mid\right)^{b}} \\
& C_{ \pm}(f ; g, h)=\int_{*} f\left(\mu_{0}, \tau_{0}\right) \frac{g\left(\mu_{1}, \tau_{1}\right)}{\left(1+\left.\left|\tau_{1}+\right| \mu_{1}\right|^{2} \mid\right)^{b}} \frac{h\left(\mu_{2}, \tau_{2}\right)}{\left(1+\left.\left|\tau_{2} \pm\right| \mu_{2}\right|^{2} \mid\right)^{b}} .
\end{aligned}
$$


Reduce (2.2) to Standard Forms. Duality and the definiton (2.1) show that $(2.2)$ is equivalent to showing

$$
\begin{gathered}
\int_{*} \frac{\left(1+\left|\mu_{0}\right|\right)^{\sigma} d\left(\mu_{0}, \tau_{0}\right)}{\left(1+\left.\left|\tau_{0}-\right| \mu_{0}\right|^{2} \mid\right)^{1-b}} \frac{\left(1+\left|\mu_{1}\right|\right)^{-s} c^{1}\left(\mu_{1}, \tau_{1}\right)}{\left(1+\left.\left|\tau_{1}-\right| \mu_{1}\right|^{2} \mid\right)^{b}} \frac{\left(1+\left|\mu_{2}\right|\right)^{-s} c^{2}\left(\mu_{2}, \tau_{2}\right)}{\left(1+\left.\left|\tau_{2}-\right| \mu_{2}\right|^{2} \mid\right)^{b}} \\
\leq C\|d\|_{L^{2}}\left\|c^{1}\right\|_{L^{2}}\left\|c^{2}\right\|_{L^{2}} .
\end{gathered}
$$

The convolution constraint $\tau_{0}+\tau_{1}+\tau_{2}=0$ and the triangle inequality imply

$$
\max \left(\left.\left|\tau_{0}-\right| \mu_{0}\right|^{2}|,| \tau_{1}-\left|\mu_{1}\right|^{2}|,| \tau_{2}-\left|\mu_{2}\right|^{2} \mid\right) \gtrsim\left|\mu_{0}\right|^{2}+\left|\mu_{1}\right|^{2}+\left|\mu_{2}\right|^{2} .
$$

We study the contribution to the left-side of (2.5) arising from the region $\left\{\left|\mu_{0}\right| \sim\right.$ $\left.M_{0}\right\},\left\{\left|\mu_{1}\right| \sim M_{1}\right\},\left\{\left|\mu_{2}\right| \sim M_{2}\right\}$ for dyadic $M_{0}, M_{1}, M_{2}$ in the three cases of (2.6) when all of the $M_{i} \geq 1$. In case the 0 -denominator is the max, we note that $\left.\left|\tau_{0}-\right| \mu_{0}\right|^{2} \mid \gtrsim\left(M^{*}\right)^{2}$ and need to estimate, after a change of sign of all the variables appearing in the integral,

$$
\frac{\left(1 \vee M_{0}\right)^{\sigma}\left(1 \vee M_{1}\right)^{-s}\left(1 \vee M_{2}\right)^{-s}}{\left(M^{*}\right)^{2(1-b)}} C_{+}\left(d_{M_{0}} ; c_{M_{1}}^{1}, c_{M_{2}}^{2}\right)
$$

and then sum over dyadic $1 \leq M_{0}, M_{1}, M_{2}$. When the 1-denominator is the max, after borrowing a bit from the 1-denominator to replace $1-b$ by $b$ on the 0 denominator, we encounter

$$
\frac{\left(1 \vee M_{0}\right)^{\sigma}\left(1 \vee M_{1}\right)^{-s}\left(1 \vee M_{2}\right)^{-s}}{\left(M^{*}\right)^{2(1-b)}} C_{+}\left(c_{M_{1}}^{1} ; d_{M_{0}}, c_{M_{2}}^{2}\right)
$$

and a similar expression arises in the final case.

Remark 1 . The low frequency cases $\left(M_{i} \leq 1\right)$ will be treated separately below using (2.5) directly.

Reduce (2.3) to Standard Forms. The estimate (2.3) is equivalent to showing

$$
\begin{gathered}
\int_{*} \frac{\left(1+\left|\mu_{0}\right|\right)^{\sigma} d\left(\mu_{0}, \tau_{0}\right)}{\left(1+\left.\left|\tau_{0}-\right| \mu_{0}\right|^{2} \mid\right)^{1-b}} \frac{\left(1+\left|\mu_{1}\right|\right)^{-s} c^{1}\left(\mu_{1}, \tau_{1}\right)}{\left(1+\left.\left|\tau_{1}+\right| \mu_{1}\right|^{2} \mid\right)^{b}} \frac{\left(1+\left|\mu_{2}\right|\right)^{-s} c^{2}\left(\mu_{2}, \tau_{2}\right)}{\left(1+\left.\left|\tau_{2}+\right| \mu_{2}\right|^{2} \mid\right)^{b}} \\
\leq C\|d\|_{L^{2}}\left\|c^{1}\right\|_{L^{2}}\left\|c^{2}\right\|_{L^{2}} .
\end{gathered}
$$

Note that

$$
\max \left(\left.\left|\tau_{0}-\right| \mu_{0}\right|^{2}|,| \tau_{1}+\left|\mu_{1}\right|^{2}|,| \tau_{2}+\left|\mu_{2}\right|^{2} \mid\right) \gtrsim\left|\mu_{1}\right|\left|\mu_{2}\right|\left|\cos \alpha_{12}\right| .
$$

The contribution from $\left\{\left|\mu_{0}\right| \sim M_{0}\right\},\left\{\left|\mu_{1}\right| \sim M_{1}\right\},\left\{\left|\mu_{2}\right| \sim M_{2}\right\}$ in case the 0denominator is the max is

$$
\frac{\left(1 \vee M_{0}\right)^{\sigma}\left(1 \vee M_{1}\right)^{-s}\left(1 \vee M_{2}\right)^{-s}}{M_{1}^{1-b} M_{2}^{1-b}} A_{+}\left(d_{M_{0}} ; c_{M_{1}}^{1}, c_{M_{2}}^{2}\right) .
$$

In case $\left.\left|\tau_{1}+\right| \mu_{1}\right|^{2} \mid$ is the max, the contribution is given by

$$
\frac{\left(1 \vee M_{0}\right)^{\sigma}\left(1 \vee M_{1}\right)^{-s}\left(1 \vee M_{2}\right)^{-s}}{M_{1}^{1-b} M_{2}^{1-b}} B_{-}\left(c_{M_{1}}^{1} ; d_{M_{0}}, c_{M_{2}}^{2}\right),
$$

and the final case leads to

$$
\frac{\left(1 \vee M_{0}\right)^{\sigma}\left(1 \vee M_{1}\right)^{-s}\left(1 \vee M_{2}\right)^{-s}}{M_{1}^{1-b} M_{2}^{1-b}} B_{-}\left(c_{M_{2}}^{2} ; d_{M_{0}}, c_{M_{1}}^{1}\right) .
$$


Remark 2 . Some low frequency cases $\left(M_{i} \leq 1\right)$ will be treated separately below using (2.9) instead of the reductions (2.11), (2.12) and (2.13).

Reduce (2.4) to Standard Forms. The $\bar{u} v$ estimate (2.4) is equivalent to showing

$$
\begin{gathered}
\int_{*} \frac{\left(1+\left|\mu_{0}\right|\right)^{\sigma} d\left(\mu_{0}, \tau_{0}\right)}{\left(1+\left.\left|\tau_{0}-\right| \mu_{0}\right|^{2} \mid\right)^{1-b}} \frac{\left(1+\left|\mu_{1}\right|\right)^{-s} c^{1}\left(\mu_{1}, \tau_{1}\right)}{\left(1+\left.\left|\tau_{1}-\right| \mu_{1}\right|^{2} \mid\right)^{b}} \frac{\left(1+\left|\mu_{2}\right|\right)^{-s} c^{2}\left(\mu_{2}, \tau_{2}\right)}{\left(1+\left.\left|\tau_{2}+\right| \mu_{2}\right|^{2} \mid\right)^{b}} \\
\leq C\|d\|_{L^{2}}\left\|c^{1}\right\|_{L^{2}}\left\|c^{2}\right\|_{L^{2}} .
\end{gathered}
$$

We have

$$
\max \left(\left.\left|\tau_{0}-\right| \mu_{0}\right|^{2}|,| \tau_{1}-\left|\mu_{1}\right|^{2}|,| \tau_{2}+\left|\mu_{2}\right|^{2} \mid\right) \gtrsim\left|\mu_{0}\right|\left|\mu_{1}\right|\left|\cos \alpha_{01}\right| .
$$

The $0,1,2$ cases of the maximum lead (respectively) to the following bounds on the contribution arising from $\left\{\left|\mu_{i}\right| \sim M_{i}\right\}$ :

$$
\begin{aligned}
& \frac{\left(1 \vee M_{0}\right)^{\sigma}\left(1 \vee M_{1}\right)^{-s}\left(1 \vee M_{2}\right)^{-s}}{M_{0}^{1-b} M_{1}^{1-b}} B_{-}\left(d_{M_{0}} ; c_{M_{2}}^{2}, c_{M_{1}}^{1}\right), \\
& \frac{\left(1 \vee M_{0}\right)^{\sigma}\left(1 \vee M_{1}\right)^{-s}\left(1 \vee M_{2}\right)^{-s}}{M_{0}^{1-b} M_{1}^{1-b}} B_{-}\left(c_{M_{1}}^{1} ; c_{M_{2}}^{2}, d_{M_{0}}\right),
\end{aligned}
$$

and

$$
\frac{\left(1 \vee M_{0}\right)^{\sigma}\left(1 \vee M_{1}\right)^{-s}\left(1 \vee M_{2}\right)^{-s}}{M_{0}^{1-b} M_{1}^{1-b}} A_{+}\left(c_{M_{2}}^{2} ; d_{M_{0}}, c_{M_{1}}^{1}\right) .
$$

Remark 3. Some low frequency cases will be treated separately below using (2.14) instead of the reductions (2.16), (2.17) and (2.18).

Estimates for Standard Trilinear Forms. Apart from certain low frequency cases, the preceding discussion reduced the study of the bilinear estimates (2.2), (2.3), (2.4) to proving bounds on the trilinear forms $A_{+}, B_{-}$and $C_{+}$when applied to functions supported on dyadic shells in the spatial frequency variable. We present some lemmas establishing such bounds and then return to the expressions above to complete the proof of the bilinear estimates.

Lemma 1. The following estimate holds,

$$
\left|C_{+}\left(f_{M_{0}} ; g_{M_{1}}, h_{M_{2}}\right)\right| \leq\left(\frac{M_{*}}{M^{*}}\right)^{\frac{1}{2}}\left\|f_{M_{0}}\right\|_{L^{2}}\left\|g_{M_{1}}\right\|_{L^{2}}\left\|h_{M_{2}}\right\|_{L^{2}},
$$

when $M^{*}>1$ and $M_{*}$ is arbitrary. We also have

$$
\left|C_{-}\left(f_{M_{0}} ; g_{M_{1}}, h_{M_{2}}\right)\right| \leq\left(\frac{M_{1} \wedge M_{2}}{M_{1} \vee M_{2}}\right)^{\frac{1}{2}}\left\|f_{M_{0}}\right\|_{L^{2}}\left\|g_{M_{1}}\right\|_{L^{2}}\left\|h_{M_{2}}\right\|_{L^{2}} .
$$

Proof. Recall that

$$
C_{ \pm}(f ; g, h)=\int_{*} f\left(\mu_{0}, \tau_{0}\right) \frac{g\left(\mu_{1}, \tau_{1}\right)}{\left(1+\left.\left|\tau_{1}+\right| \mu_{1}\right|^{2} \mid\right)^{b}} \frac{h\left(\mu_{2}, \tau_{2}\right)}{\left(1+\left.\left|\tau_{2} \pm\right| \mu_{2}\right|^{2} \mid\right)^{b}} .
$$


Parabolic Level Set Decomposition. Cut the functions $g, h$ into parabolic level sets by writing $g=\sum_{l_{1} \in \mathbb{Z}} g_{l_{1}}, h=\sum_{l_{2} \in \mathbb{Z}} h_{l_{2}}$ where $g_{l_{1}}=g \chi_{\left\{\left(\tau_{1}+\left|\mu_{1}\right|^{2}\right)=l_{1}+O(1)\right\}}$ and $h_{l_{2}}=h \chi_{\left\{\left(\tau_{2} \pm\left|\mu_{2}\right|^{2}\right)=l_{2}+O(1)\right\}}$. Matters collapse to controlling

$$
\begin{aligned}
\sum_{l_{1}, l_{2}} & \frac{1}{\left(1+\left|l_{1}\right|\right)^{b}\left(1+\left|l_{2}\right|\right)^{b}} \int_{\left\{\left|\theta_{i}\right|<O(1)\right\}} \int f\left(-\mu_{1}-\mu_{2},-\left|\mu_{1}\right|^{2} \pm\left|\mu_{2}\right|^{2}-\theta_{1}-\theta_{2}-l_{1}-l_{2}\right) \\
& \times g_{l_{1}}\left(\mu_{1},-\left|\mu_{1}\right|^{2}+l_{1}+\theta_{1}\right) h_{l_{2}}\left(\mu_{2}, \pm\left|\mu_{2}\right|^{2}+l_{2}+\theta_{2}\right) d \mu_{1} d \mu_{2} d \theta_{1} d \theta_{2}
\end{aligned}
$$

Since $b>\frac{1}{2}$, Cauchy-Schwarz in $l_{1}, l_{2}$ establishes (2.19) and (2.20) if we prove

$$
\begin{aligned}
\int_{\theta_{i}=O(1)} & \int f_{M_{0}}\left(-\mu_{1}-\mu_{2},-\left|\mu_{1}\right|^{2}-\left|\mu_{2}\right|^{2}+\theta_{1}+\theta_{2}\right) \\
& \times g_{M_{1}}\left(\mu_{1},-\left|\mu_{1}\right|^{2}+\theta_{1}\right) h_{M_{2}}\left(\mu_{2},-\left|\mu_{2}\right|^{2}+\theta_{2}\right) d \mu_{i} d \theta_{i} \\
\leq & \left(\frac{M_{*}}{M^{*}}\right)^{\frac{1}{2}}\left\|f_{M_{0}}\right\|_{L^{2}}\left\|g_{M_{1}}\right\|_{L^{2}}\left\|h_{M_{2}}\right\|_{L^{2}},
\end{aligned}
$$

and

$$
\begin{aligned}
\int_{\theta_{i}=O(1)} & \int f_{M_{0}}\left(-\mu_{1}-\mu_{2},-\left|\mu_{1}\right|^{2}+\left|\mu_{2}\right|^{2}+\theta_{1}+\theta_{2}\right) \\
& \times g_{M_{1}}\left(\mu_{1},-\left|\mu_{1}\right|^{2}+\theta_{1}\right) h_{M_{2}}\left(\mu_{2},+\left|\mu_{2}\right|^{2}+\theta_{2}\right) d \mu_{i} d \theta_{i} \\
\leq & \left(\frac{M_{1} \wedge M_{2}}{M_{1} \vee M_{2}}\right)^{\frac{1}{2}}\left\|f_{M_{0}}\right\|_{L^{2}}\left\|g_{M_{1}}\right\|_{L^{2}}\left\|h_{M_{2}}\right\|_{L^{2}},
\end{aligned}
$$

respectively.

We focus our attention on (2.21). Since $\mu_{1}$ and $\mu_{2}$ appear symmetrically, we may assume $M_{1} \gtrsim M_{2}$ and consider the three cases $M_{0} \ll M_{1} \Longrightarrow M_{1} \sim M_{2}$ and $M_{0} \sim M_{1} \gg M_{2}, M_{0} \sim M_{1} \sim M_{2}$.

Case 1. $M_{0} \ll M_{1} \sim M_{2}$.

Since $\left|\mu_{1}+\mu_{2}\right|=\left|\mu_{0}\right| \sim M_{0}$, we know that $\left|\mu_{2}-\mu_{1}\right| \sim M_{1}$. We may assume $\left|\mu_{2}^{2}-\mu_{1}^{2}\right| \sim M_{1}$ since a modification of what follows applies in the alternative case $\left|\mu_{2}^{2}-\mu_{1}^{2}\right| \ll M_{1} \Longrightarrow\left|\mu_{2}^{1}-\mu_{1}^{1}\right| \sim M_{1}$. This modification is described below. We decompose $\left\{\left|\mu_{1}\right| \sim M_{1}\right\}$ into disjoint cubes $Q_{i}$ of side $M_{0}$ and similarly decompse $\left\{\left|\mu_{2}\right| \sim M_{2}\right\}$ into cubes $Q_{j_{2}}$ of side $M_{0}$. Since $\mu_{0}+\mu_{1}+\mu_{2}=0, Q_{j_{2}}$ is uniquely determined by $Q_{j_{1}}$ which we indicate by writing $j_{2}=j_{2}\left(j_{1}\right)$. For fixed $\theta_{1}, \theta_{2}$, we change variables $u=-\mu_{1}-\mu_{2}, v=-\left|\mu_{1}\right|^{2}-\left|\mu_{2}\right|^{2}+\theta_{1}+\theta_{2}$, $d u d v d \mu_{2}^{1}=J d \mu_{1}^{1} d \mu_{1}^{2} d \mu_{2}^{2} d \mu_{2}^{1}$. A calculation shows $J=2\left|\mu_{2}^{2}-\mu_{1}^{2}\right|$ which is of size $M_{1}$ by assumption. The $\mu_{1} \mu_{2}$-integration in (2.21) may be re-expressed as

$$
\sum_{Q_{j_{1}}} \int_{\mu_{2}^{1} \in \pi^{1} Q_{j_{2}\left(j_{1}\right)}} \int f_{M_{0}}(u, v) H\left(u, v, \mu_{2}^{1}\right) d u d v d \mu_{2}^{1}
$$

where $\pi^{1}$ is the projection onto the first component and

$$
H\left(u, v, \mu_{2}^{1}\right)=\frac{g_{M_{1}, Q_{j_{1}}}\left(\mu_{1},-\left|\mu_{1}\right|^{2}+\theta_{1}\right) h_{M_{2}, Q_{j_{2}\left(j_{1}\right)}}\left(\mu_{2},-\left|\mu_{2}\right|^{2}+\theta_{2}\right)}{J} .
$$


Cauchy-Schwarz in $u, v$ followed by Cauchy-Schwarz in $\mu_{2}^{1}$ and changing back to the original variables leads to

$$
\begin{aligned}
\sum_{Q_{j_{1}}} & \left(\frac{M_{0}}{M_{1}}\right)^{\frac{1}{2}}\left\|f_{M_{0}}\right\|_{L^{2}}\left(\int\left|g_{M_{1}, Q_{j_{1}}}\left(\mu_{1},-\left|\mu_{1}\right|^{2}+\theta_{1}\right)\right|^{2} d \mu_{1}\right)^{\frac{1}{2}} \\
& \times\left(\int\left|h_{M_{2}, Q_{j_{2}}}\left(\mu_{2},-\left|\mu_{2}\right|^{2}+\theta_{2}\right)\right|^{2} d \mu_{2}\right)^{\frac{1}{2}}
\end{aligned}
$$

as the desired upper bound on the inner integral in (2.21). Cauchy-Schwarz in $Q_{j_{1}}$ and $\theta_{i}$ finishes off this case.

In case $\left|\mu_{2}^{2}-\mu_{1}^{2}\right| \ll M_{1} \Longrightarrow\left|\mu_{2}^{1}-\mu_{1}^{1}\right| \sim M_{1}$, we modify the change of variable by using the same $u, v$ as above but $d u d v d \mu_{2}^{2}=J d \mu_{1}^{1} d \mu_{1}^{2} d \mu_{2}^{1} d \mu_{2}^{2}$. Upon calculating, we find $J=2\left|\mu_{1}^{1}-\mu_{2}^{1}\right|$ which is of size $M_{1}$. Therefore, the Cauchy-Schwarz and change of variables argument used previously leads to the same result here.

Case 2. $M_{0} \sim M_{1} \gg M_{2}$.

Since $M_{2}<M_{1}$, the change of variable used above satisfies, assuming $\left|\mu_{1}^{2}\right| \gtrsim M_{1}$, $J \sim M_{1}$. The situation when $\mu_{1}^{1}$ is the big component of $\mu_{1}$ is handled similarly. We decompose $\left\{\left|\mu_{0}\right| \sim M_{0}\right\}$ into cubes $Q_{j_{0}}$ of side $M_{2}$ and $\left\{\left|\mu_{1}\right| \sim M_{1}\right\}$ into cubes $Q_{j_{1}}$ also of side $M_{2}$. Again, $j_{0}=j_{0}\left(j_{2}\right)$ and an imitation of the Case 1 argument yields the upper bound

$$
\left(\frac{M_{2}}{M_{1}}\right)^{\frac{1}{2}}\left\|f_{M_{0}}\right\|_{L^{2}}\left\|g_{M_{1}}\right\|_{L^{2}}\left\|h_{M_{2}}\right\|_{L^{2}}
$$

Case 3. $M_{0} \sim M_{1} \sim M_{2}$.

Here, we have $M_{*} \sim M^{*}$ and return to the expression

$$
\int_{*} f\left(\mu_{0}, \tau_{0}\right) \frac{g\left(\mu_{1}, \tau_{1}\right)}{\left(1+\left.\left|\tau_{1}+\right| \mu_{1}\right|^{2} \mid\right)^{b}} \frac{h\left(\mu_{2}, \tau_{2}\right)}{\left(1+\left.\left|\tau_{2}+\right| \mu_{2}\right|^{2} \mid\right)^{b}} .
$$

The desired estimate then follows in this case using a familiar argument exploiting the Strichartz estimate

$$
\left\|\iint \frac{a(k, \lambda)}{\left(1+\left.|\lambda \pm| k\right|^{2}\right)^{b}} e^{i(k x+\lambda t)} d k d \lambda\right\|_{L_{x t}^{4}} \leq C\|a\|_{L^{2}} .
$$

This completes the proof of (2.21).

We now turn our attention to (2.22) and break the analysis into four cases:

1. $M_{0} \sim M_{1} \gg M_{2}$,

2. $M_{0} \sim M_{2} \gg M_{1}$,

3. $M_{1} \sim M_{2} \gg M_{0}$ and

4. $M_{1} \sim M_{2} \sim M_{0}$.

In cases 1 and 2, we bust the largest shells into cubes of the smallest scale and make a change of variable. Cases 3 and 4 follow directly from the Strichartz inequality since $M_{1} \wedge M_{2} \sim M_{1} \vee M_{2}$.

Case 1. $M_{0} \sim M_{1} \gg M_{2}$.

Bust up the $M_{0}$ and $M_{1}$ shells into cubes $Q_{j_{0}}, Q_{j_{2}}$ (respectively) of side $M_{2}$. Rotate coordinates such that $\left|\mu_{1}^{2}\right| \sim\left|\mu_{1}\right|$. We change variables $u=-\mu_{1}-\mu_{2}$, $v=-\left|\mu_{1}\right|^{2}+\left|\mu_{2}\right|^{2}+\theta_{1}+\theta_{2}$ with $d u d v d \mu_{2}^{1}=J d \mu_{1}^{1} d \mu_{1}^{2} d \mu_{2}^{2} d \mu_{2}^{1}$. A calculation 
shows $J=2\left|\mu_{1}^{2}-\mu_{2}^{2}\right|$ which may be assumed to have size $\sim M_{1}$. Cauchy-Schwarz applications lead to the bound

$$
\left(\frac{M_{2}}{M_{1}}\right)^{\frac{1}{2}}\left\|f_{M_{0}}\right\|_{L^{2}}\left\|g_{M_{1}}\right\|_{L^{2}}\left\|h_{M_{2}}\right\|_{L^{2}}
$$

as claimed.

Case 2. $M_{0} \sim M_{2} \gg M_{1}$.

The change of variable used in Case 1 above now has $J \sim M_{2}$, assuming $\left|\mu_{2}^{2}\right| \sim$ $M_{2}$. We break the $M_{0}, M_{2}$ shells into $M_{1}$-sized cubes and imitate the preceding argument.

As mentioned above, Cases 3 and 4 follow from (2.24).

\section{Lemma 2.}

$$
\left|A_{+}\left(f_{M_{0}} ; g_{M_{1}}, h_{M_{2}}\right)\right| \leq C\left\|f_{M_{0}}\right\|_{L^{2}}\left\|g_{M_{1}}\right\|_{L^{2}}\left\|h_{M_{2}}\right\|_{L^{2}} .
$$

Proof. Recall that

$$
A_{+}(f ; g, h)=\int_{*} \frac{f\left(\mu_{0}, \tau_{0}\right)}{\left|\cos \alpha_{12}\right|^{1-b}} \frac{g\left(\mu_{1}, \tau_{1}\right)}{\left(1+\left.\left|\tau_{1}+\right| \mu_{1}\right|^{2} \mid\right)^{b}} \frac{h\left(\mu_{2}, \tau_{2}\right)}{\left(1+\left.\left|\tau_{2}+\right| \mu_{2}\right|^{2} \mid\right)^{b}} .
$$

The symmetric appearance of $\mu_{1}, \mu_{2}$ allows us to assume $M_{1} \geq M_{2}$. In case $\left|\cos \alpha_{12}\right|>\delta>0$, we reduce to $C_{+}\left(f_{M_{0}} ; g_{M_{1}}, h_{M_{2}}\right)$ which was appropriately estimated in Lemma 10 We may assume $\left|\cos \alpha_{12}\right|$ is small and, therefore, that $\mu_{1}$ is almost perpendicular to $\mu_{2}$.

Consider the contribution arising from the region where $\left|\cos \alpha_{12}\right| \sim \nu \ll 1$ for $\nu$ dyadic. A parabolic level set decomposition using $b>\frac{1}{2}$ reduces matters to considering

$$
\begin{array}{r}
\frac{1}{\nu^{1-b}} \int_{\theta_{i}=O(1)} \int_{\substack{\left\{\left|\cos \alpha_{12}\right| \sim \nu\right\} \\
\times g_{M_{1}}\left(\mu_{1},-\left|\mu_{1}\right|^{2}+\theta_{1}\right) h_{M_{2}}\left(\mu_{2},-\left|\mu_{2}\right|^{2}+\theta_{2}\right) d \mu_{i} d \theta_{i} .}} f_{M_{0}}\left(-\mu_{1}-\mu_{2},+\left|\mu_{1}\right|^{2}+\left|\mu_{2}\right|^{2}+\theta_{1}+\theta_{2}\right)
\end{array}
$$

The small cosine level set suggests decomposing the $M_{1}$ and $M_{2}$ shells into disjoint "pie slices" of angular aperture $\nu$. Write for $i=1,2,\left\{\left|\mu_{i}\right| \sim M_{i}\right\}=\bigcup_{j_{i}} A_{j_{i}}$ with $A_{j_{i}}=\left\{k_{i}: k_{i}=\left|k_{i}\right| e^{i \theta},\left|\theta-j_{i} \nu 2 \pi\right|<O(\nu)\right\}$ pairwise disjoint. The cosine level set condition $\left|\cos \alpha_{12}\right| \sim \nu$ forces $A_{j_{2}}=A_{j_{2}\left(j_{1}\right)}$ for nonzero contribution. We replace $g_{M_{1}}$ by $g_{M_{1}, A_{j_{1}}}$ and $h_{M_{2}}$ by $h_{M_{2}, A_{j_{2}}}$ in (2.26) and must sum over $j_{1}$. For fixed $j_{1}$, we can rotate to ensure $A_{j_{2}\left(j_{1}\right)}$ is bisected by the $\mu_{2}^{2}$ axis. Note then that $\left|\pi^{1}\left(A_{j_{2}}\right)\right| \sim \nu M_{2}$ where we have again used $\pi^{1}$ to denote the projection onto the first coordinate. We may assume that $A_{j_{1}}$ lies (nearly) along the positive $\mu_{1}^{1}$ axis.

We apply the change of variable used in Case 1 of the proof of Lemma[1. Since the Jacobian $J=\left|2\left(\mu_{1}^{2}-\mu_{2}^{2}\right)\right|$, the arrangement of the "pie slices" shows $J \sim$ $M_{2}$.11 The "extra integration" along $\mu_{2}^{1}$ takes place along $\pi^{1}\left(A_{j_{2}}\right)$ which is of size $\nu M_{2}$. Therefore, the change of variable and Cauchy-Schwarz show the fixed $j_{1}$ contribution to (2.26) is bounded by

$$
\frac{1}{\nu^{1-b}} \frac{\left(\nu M_{2}\right)^{\frac{1}{2}}}{M_{2}^{\frac{1}{2}}}\left\|f_{M_{0}}\right\|_{L^{2}}\left\|g_{M_{1}, A_{j_{1}}}\right\|_{L^{2}}\left\|h_{M_{2}, A_{j_{2}\left(j_{1}\right)}}\right\|_{L^{2}}
$$

\footnotetext{
${ }^{1}$ There is the possibility that $\nu M_{1} \gtrsim M_{2}$ in which case $\mu_{1}^{2}-\mu_{2}^{2}$ may be very small. If this occurs, a change of variable with $\mu_{2}^{2}$ playing the "extra integration" role leads to the same conclusion.
} 
and Cauchy-Schwarz allows us to sum in $j_{1}$. Finally, note that $\nu^{\frac{1}{2}-(1-b)}=\nu^{\epsilon}, \epsilon>0$, so we can sum over small dyadic levels of the cosine completing the proof.

\section{Lemma 3.}

$$
B_{-}\left(f_{M_{0}} ; g_{M_{1}}, h_{M_{2}}\right) \leq C\left\|f_{M_{0}}\right\|_{L^{2}}\left\|g_{M_{1}}\right\|_{L^{2}}\left\|h_{M_{2}}\right\|_{L^{2}} .
$$

Proof. Recall that

$$
B_{-}(f ; g, h)=\int_{*} \frac{f\left(\mu_{0}, \tau_{0}\right)}{\left|\cos \alpha_{02}\right|^{1-b}} \frac{g\left(\mu_{1}, \tau_{1}\right)}{\left(1+\left.\left|\tau_{1}+\right| \mu_{1}\right|^{2} \mid\right)^{b}} \frac{h\left(\mu_{2}, \tau_{2}\right)}{\left(1+\left.\left|\tau_{2}-\right| \mu_{2}\right|^{2} \mid\right)^{b}},
$$

A parabolic level set decomposition collapses the issue to controlling

$$
\begin{aligned}
& \int_{\theta_{i}=O(1)} \int \frac{f_{M_{0}}\left(-\mu_{1}-\mu_{2},-\left|\mu_{1}\right|^{2}+\left|\mu_{2}\right|^{2}+\theta_{1}+\theta_{2}\right)}{\left|\cos \alpha_{02}\right|^{1-b}} \\
& \quad \times g_{M_{1}}\left(\mu_{1},-\left|\mu_{1}\right|^{2}+\theta_{1}\right) h_{M_{2}}\left(\mu_{2},\left|\mu_{2}\right|^{2}+\theta_{2}\right) d \mu_{i} d \theta_{i} .
\end{aligned}
$$

In case $\left|\cos \alpha_{02}\right|>\delta>0$, we encounter the previously estimated $C_{-}$expression. We may therefore assume the cosine term is small. Consider the contribution arising from $\left|\cos \alpha_{02}\right| \sim \nu \ll 1, \nu$ dyadic.

Case 1. $\nu M_{0} \leq M_{2}$. As in the proof of Lemma 2 2 , we cut the $M_{0}$ and $M_{2}$ shells into disjoint "pie slices" $A_{j_{0}\left(j_{2}\right)}$ and $A_{j_{2}}$. We have $j_{0}\left(j_{2}\right)$ using the cosine level set condition and $\mu_{0}+\mu_{1}+\mu_{2}=0$.

For fixed $j_{2}$, rotate such that $A_{j_{0}\left(j_{2}\right)}$ is bisected by the positive $\mu_{0}^{2}$ axis. Note that $A_{j_{0}\left(j_{2}\right)}$ is essentially a $\nu M_{0} \times M_{0}$ rectangle. We may assume $A_{j_{2}}$ is almost along the positive $\mu_{2}^{1}$ axis. Decompose $A_{j_{2}}$ and the $M_{1}$ shell into disjoint translates of the smallest rectangle containing $A_{j_{0}\left(j_{2}\right)}$. Therefore, we can write $\left\{\left|\mu_{1}\right| \sim M_{1}\right\}=\bigcup R_{i_{1}}$ and $A_{j_{2}}=\bigcup R_{i_{2}}$. Since $\mu_{0}+\mu_{1}+\mu_{2}=0$, we have $i_{1}=i_{1}\left(i_{2}\right)$. Note that this decomposition cuts $A_{j_{2}}$ into disjoint rectangles of size $\nu M_{0}$ along the $\mu_{2}^{1}$ coordinate axis and of size $\left(\nu M_{2} \wedge M_{0}\right)$ along the $\mu_{2}^{2}$ axis.

The fixed $j_{2}$ contribution to the $\left|\cos \alpha_{02}\right| \sim \nu$ contribution to (2.28) is estimated by

$$
\begin{aligned}
& \frac{1}{\nu^{1-b}} \sum_{i_{2}} \int_{\theta_{i}=O(1)} \int f_{M_{0}, A_{j_{0}}}\left(\mu_{0},-\left|\mu_{1}+\mu_{2}\right|^{2}+\left|\mu_{2}\right|^{2}+\theta_{1}+\theta_{2}\right) \\
& \quad \times g_{M_{1}, R_{i_{1}\left(i_{2}\right)}}\left(-\mu_{0}-\mu_{2},-\left|\mu_{0}+\mu_{2}\right|^{2}+\theta_{2}\right) h_{M_{2}, R_{i_{2}}}\left(\mu_{2},\left|\mu_{2}\right|^{2}+\theta_{2}\right) d \mu_{i} d \theta_{i} .
\end{aligned}
$$

The change of variables $u=\mu_{0}, v=\left|\mu_{0}+\mu_{2}\right|^{2}-\left|\mu_{2}\right|^{2}+\theta_{1}+\theta_{2}, d u d v d \mu_{2}^{1}=$ $J d \mu_{0}^{1} d \mu_{0}^{2} d \mu_{2}^{2} d \mu_{2}^{1}$ has $J=2\left|\mu_{0}^{2}\right| \sim M_{0}$. Note that $\left|\pi^{1}\left(R_{i_{2}}\right)\right| \sim \nu M_{0}$ so the "extra integration" in $\mu_{2}^{1}$ leads to the factor $\left(\nu M_{0}\right)^{\frac{1}{2}}$. Familiar Cauchy-Schwarz applications eventually give

$$
\frac{1}{\nu^{1-b}} \sum_{i_{2}}\left(\frac{\nu M_{0}}{M_{0}}\right)^{\frac{1}{2}}\left\|f_{M_{0}, A_{j_{0}}}\right\|_{L^{2}}\left\|g_{M_{1}, R_{i_{1}\left(i_{2}\right)}}\right\|_{L^{2}}\left\|h_{M_{2}, R_{i_{2}}}\right\|_{L^{2}} .
$$

Applying Cauchy-Schwarz in $i_{2}$ reveals

$$
\nu^{\frac{1}{2}-(1-b)}\left\|f_{M_{0}, A_{j_{0}}}\right\|_{L^{2}}\left\|g_{M_{1}}\right\|_{L^{2}}\left\|h_{M_{2}, A_{j_{2}}}\right\|_{L^{2}} \text {. }
$$

Recalling that $j_{0}=j_{0}\left(j_{2}\right)$, we apply Cauchy-Schwarz to sum in $j_{2}$ and use $\frac{1}{2}-$ $(1-b)>0$ to sum over small dyadic levels of $\left|\cos \alpha_{02}\right|$. 
Case 2. $\nu M_{0}>M_{2}$.

We cut the same decomposition into "pie slices". We fix $j_{2}$ and rotate into the configuration considered above. The change of variables has $J \sim\left|\mu_{0}^{2}\right| \sim M_{0}$. Decompose $A_{j_{0}\left(j_{2}\right)}$ and the $M_{1}$ shell into disjoint translates of $A_{j_{2}}$. For any translate $\tau$, we have $\left|\pi^{1}\left(\tau\left(A_{j_{2}}\right)\right)\right| \sim M_{2}<\nu M_{0}$. Therefore, the "extra integration" and the Jacobian manufacture the prefactor $\left(\frac{M_{2}}{M_{0}}\right)^{\frac{1}{2}}<\left(\frac{\nu M_{0}}{M_{0}}\right)^{\frac{1}{2}}$, and orthogonality and Cauchy-Schwarz applications complete the proof.

Proof of Bilinear Estimates. The low frequency region $M_{0}, M_{1}, M_{2} \lesssim 1$ of the estimates (2.2), (2.3) and (2.4) is treated the same way. Since the terms $\left(1+\left|\mu_{i}\right|\right)^{(-s)}$ or $\left(1+\left|\mu_{i}\right|\right)^{(\sigma)}$ in the numerator are all $O(1)$, we ignore them. The remaining expression is estimated using the Strichartz estimate (2.24) using $b>\frac{1}{2}$. We may now assume throughout that $M^{*}>1$.

We turn our attention to establishing (2.2). Symmetry in $\mu_{1}, \mu_{2}$ allows us to assume $M_{1} \geq M_{2}$. In Case $1, M_{2} \ll 1 \ll M_{1} \Longrightarrow M_{1} \sim M_{0}$, an application of the $C_{+}$lemma (2.19) gives the bound $M_{1}^{\sigma-s-2(1-b)-\frac{1}{2}} M_{2}^{\frac{1}{2}}$. This is summable in dyadic $M_{1} \geq 1$ if we require $\sigma<s+\frac{1}{2}+2(1-b)$. Clearly, we have summability over dyadic $M_{2} \ll 1$. In Case $2, M_{0} \ll 1 \ll M_{1} \Longrightarrow M_{1} \sim M_{2}$; we again apply (2.19) to (2.7) and (2.8) to find $M_{1}^{-2 s-2(1-b)-\frac{1}{2}} M_{0}^{\frac{1}{2}}$ which sums over dyadic $M_{1} \gg 1$ provided $-\frac{1}{4}-(1-b)<s$ and also over dyadic $M_{0} \ll 1$. In Case $3,1 \lesssim M_{0}, M_{1}, M_{2}$ with $M_{1} \geq M_{2}$. When $1 \lesssim M_{0} \ll M_{1} \Longrightarrow M_{1} \sim M_{2}$, we are led, using (2.19) on (2.7), (2.8) to $M_{0}^{\sigma+\frac{1}{2}} M_{1}^{-2 s-2(1-b)-\frac{1}{2}}$. If $\sigma+\frac{1}{2} \geq 0$, we bound by $M_{1}^{\sigma+\frac{1}{2}-2 s-2(1-b)-\frac{1}{2}}$ and can sum using the condition $\sigma<2 s+2(1-b)$. For $\sigma+\frac{1}{2}<0$, we can sum over dyadic $M_{0} \geq 1$, and the condition $-\frac{1}{4}-(1-b)<s$ allows us to sum in $M_{1}$. In case $1 \lesssim M_{0} \sim M_{1}$ with $1 \lesssim M_{2} \leq M_{1}$, we are led to $M_{1}^{\sigma-s-2(1-b)-\frac{1}{2}} M_{2}^{-s+\frac{1}{2}}$. If $-s+\frac{1}{2}<0$, we can sum over dyadic $M_{2} \gtrsim 1$. When $-s+\frac{1}{2} \geq 0$, we can replace $M_{2}$ by $M_{1}$ and require $\sigma<2 s+2(1-b)$ to sum in $M_{1}$.

Summarizing, we have found that (2.2) is valid when $b=\frac{1}{2}+,-\frac{1}{4}-(1-b)<s$ and $\sigma<\min \left(s+\frac{1}{2}+2(1-b), 2 s+2(1-b)\right)$.

We now concentrate on establishing (2.3). Again by symmetry, $M_{1} \geq M_{2}$. The low frequency case common to (2.2), (2.3) and (2.4) treated above allows us to assume $M^{*}>1$. We first consider the situation leading to (2.11).

1. $M_{0} \ll M_{1} \Longrightarrow M_{1} \sim M_{2}$ and, moreover, $\mu_{1} \sim-\mu_{2}$ so we are certain that $\left|\cos \alpha_{12}\right|>\delta>0$ and can use the $C_{+}$estimate (2.19) instead of (2.25). This yields $\left(1 \vee M_{0}\right)^{\sigma} M_{0}^{\frac{1}{2}} M_{1}^{-2 s-2(1-b)-\frac{1}{2}}$. This is fine for $M_{0} \ll 1$ provided $-\frac{1}{4}-(1-b)<s$. For $M_{0} \gtrsim 1$, we consider two possibilities: When $\sigma+\frac{1}{2}<0$, we can ignore $M_{0}$ and are fine if $-\frac{1}{4}-(1-b)<s$; when $\sigma+\frac{1}{2} \geq 0$, we replace $M_{0}$ by $M_{1}$ and ask that $\sigma<2 s+2(1-b)$.

2. $1 \lesssim M_{2} \lesssim M_{1} \sim M_{0}$. We apply the estimate (2.25) to obtain the bound $M_{1}^{\sigma-s-(1-b)} M_{2}^{-s-(1-b)}$. Considering the two cases $s+(1-b) \leq 0, s+(1-b)>0$, one finds that $\sigma<\min \left(s+\frac{1}{2}, 2 s+2(1-b)\right)$ suffices.

3. $M_{2} \ll 1 \lesssim M_{1} \sim M_{0}$. We return to the left side of (2.9) and replace the largest denominator by 1 . The expression that remains is $M_{0}^{\sigma} M_{1}^{-s} C_{+}\left(d_{M_{0}} ; c_{M_{1}}^{1}, c_{M_{2}}^{2}\right)$ which we estimate using (2.19) and sum provided $\sigma<s+\frac{1}{2}$. The contribution (2.11) to the left side of (2.3) is appropriately bounded provided $b=\frac{1}{2}+,-\frac{1}{4}-(1-b)<s$ and $\sigma<\min \left(s+\frac{1}{2}, 2 s+2(1-b)\right)$. 
Next, we consider (2.12).

1. $M_{0} \ll M_{1} \sim M_{2} \Longrightarrow \mu_{1} \sim-\mu_{2}$ and therefore $\left|\cos \alpha_{12}\right|>\delta>0$ in $B_{-}$so we can apply the $C_{-}$estimate (2.20). This leads to $\left(1 \vee M_{0}\right)^{\sigma} M_{0}^{\frac{1}{2}} M_{1}^{-2 s-2(1-b)-\frac{1}{2}}$. If $\sigma+\frac{1}{2}<0$ and $-\frac{1}{4}-(1-b)<s$, this sums over dyadic $M_{0}, M_{1}$. When $\sigma+\frac{1}{2} \geq 0$, we replace $M_{0}$ by $M_{1}$ and impose the condition $\sigma<2 s+2(1-b)$.

2. $M_{0} \sim M_{1} \gtrsim M_{2} \gtrsim 1$. The $B_{-}$estimate (2.27) gives $M_{1}^{\sigma-s-(1-b)} M_{2}^{-s-(1-b)}$. When $-s-(1-b) \geq 0$, we replace $M_{2}$ by $M_{1}$ and require $\sigma<2 s+2(1-b)$. When $-s-(1-b)<0$, we ignore $M_{2}$ and need $\sigma<s+(1-b)$ which follows from $\sigma<s+\frac{1}{2}$ for $b=\frac{1}{2}+$.

3. $M_{2} \ll 1 \lesssim M_{1} \sim M_{0}$. We return to (2.9), replace the largest denominator by 1 and encounter $M_{0}^{\sigma} M_{1}^{-s} C_{-}\left(c_{M_{1}}^{1} ; d_{M_{0}}, c_{M_{2}}^{2}\right)$. Applying (2.20) leads to $M_{0}^{\sigma} M_{1}^{-s}\left(\frac{M_{2}}{M_{1}}\right)^{\frac{1}{2}}$ which sums over small dyadic $M_{2}$ and also over $M_{1} \sim M_{0}$ since $\sigma<s+\frac{1}{2}$.

The treatment of (2.13) is similar apart from one subcase. When $M_{2}<1 \leq$ $M_{1}=M_{0}$, we return to (2.9) and replace $\left(1+\left.\left|\tau_{0}-\right| \mu_{0}\right|^{2} \mid\right)^{1-b}$ by 1 leaving $M_{0}^{\sigma} M_{1}^{-s}$ $C_{+}\left(d_{M_{0}} ; c_{M_{2}}^{2}, c_{M_{1}}^{1}\right)$ which we estimate using (2.19).

Therefore, (2.3) holds if the parameters satisfy $b=\frac{1}{2}+,-\frac{1}{4}-(1-b)<s$ and $\sigma<\min \left(s+\frac{1}{2}, 2 s+2(1-b)\right)$.

We now prove (2.4).

1. $1 \lesssim M_{0} \ll M_{1} \sim M_{2}$. We have $M_{0}^{\sigma-(1-b)} M_{1}^{-2 s-(1-b)}$. If $\sigma-(1-b) \geq 0$, replace $M_{0}$ by $M_{1}$ and require $\sigma<2 s+2(1-b)$. If $\sigma-(1-b)<0$, ignore $M_{0}$ and require $-\frac{1}{2}(1-b)<s$.

2. $M_{0} \ll 1 \lesssim M_{1} \sim M_{2}$. We return to (2.14). If the largest denominator $\left(1+\left.\left|\tau_{0}-\right| \mu_{0}\right|^{2} \mid\right)>M_{1}$, we apply (2.20) and need to sum $M_{1}^{-2 s} \frac{1}{M_{1}^{1-6}}$ which is fine. If $\left(1+\left.\left|\tau_{0}-\right| \mu_{0}\right|^{2} \mid\right)<M_{1}$, we pay a small penalty by multiplying by $\frac{M_{1}^{\epsilon}}{\left(1+\left.\left|\tau_{0}-\right| \mu_{0}\right|^{2} \mid\right)^{\epsilon}}>$ 1. This leads to

$$
M_{1}^{-2 s} M_{1}^{\epsilon} \int_{*} \frac{d\left(\mu_{0}, \tau_{0}\right)}{\left(1+\left.\left|\tau_{0}-\right| \mu_{0}\right|^{2} \mid\right)^{b}} \frac{c^{1}\left(\mu_{1}, \tau_{1}\right)}{\left(1+\left.\left|\tau_{1}-\right| \mu_{1}\right|^{2} \mid\right)^{b}} \frac{c^{2}\left(\mu_{2}, \tau_{2}\right)}{\left(1+\left.\left|\tau_{2}+\right| \mu_{2}\right|^{2} \mid\right)^{b}} .
$$

Since $b=\frac{1}{2}+$, we can take $\epsilon>0$ arbitrarily small. We replace $\left(1+\left.\left|\tau_{1}-\right| \mu_{1}\right|^{2} \mid\right)^{b}$ by 1 and find $M_{1}^{-2 s+\epsilon} C_{-}\left(c_{M_{1}}^{1} ; c_{M_{2}}^{2}, d_{M_{0}}\right)$. Applying (2.20) gives $M_{1}^{-2 s+\epsilon}\left(\frac{M_{0}}{M_{2}}\right)^{\frac{1}{2}}$ and summability requires $-2 s+\epsilon-\frac{1}{2}<0$ or $-\frac{1}{4}<s$ which follows from the assumption $-\frac{1}{2}(1-b)<s$ with $b=\frac{1}{2}+$.

3. $M_{1} \ll 1 \lesssim M_{2} \sim M_{0}$. We forget the largest denominator in (2.14) and find $M_{0}^{\sigma} M_{2}^{-s} C_{-}\left(d_{M_{0}} ; c_{M_{2}}^{2}, c_{M_{1}}^{1}\right)$ which is fine using (2.20).

4. $1 \ll M_{1} \lesssim M_{0} \sim M_{2}$. We have $M_{2}^{\sigma-s-(1-b)} M_{1}^{-s-(1-b)}$. If $-s-(1-b)<0$, ignore $M_{1}$ and require $\sigma<s+(1-b)$ which follows from $\sigma<s+\frac{1}{2}$ for $b=\frac{1}{2}+$. If $-s-(1-b) \geq 0$, replace $M_{1}$ by $M_{2}$ and require $\sigma<2 s+2(1-b)$.

5. $M_{2} \ll M_{0} \sim M_{1} \Longrightarrow \mu_{0} \sim-\mu_{1}$ and therefore $\left|\cos \alpha_{01}\right|>\delta>0$. Applying (2.20) leads to $M_{1}^{\sigma-s-2(1-b)-\frac{1}{2}}\left(1 \vee M_{2}\right)^{-s} M_{2}^{\frac{1}{2}}$. If $-s+\frac{1}{2} \geq 0$, replace $M_{2}$ by $M_{1}$ and require $\sigma<2 s+2(1-b)$. If $-s+\frac{1}{2}<0$, we can ignore $M_{2}$ and easily sum in $M_{1}$. 
6. $M_{0} \sim M_{1} \sim M_{2}$. Apply the $B_{-}$estimate (2.27) to (2.16) yielding the condition $\sigma<2 s+2(1-b)$. The cases (2.17) and (2.18) can be checked in a similar way.

In conclusion, the estimate (2.4) holds provided $b=\frac{1}{2}+,-\frac{1}{2}(1-b)<s$ and $\sigma<\min \left(s+\frac{1}{2}, 2 s+2(1-b)\right)$.

Examples Demonstrating Necessity of Parameter Restrictions. We begin by considering the estimate (2.2). Define the set $R_{N}=\{(k, \lambda):|k-(N, 0)| \leq$ $\left.1,\left.|\lambda+| k\right|^{2} \mid \leq 1\right\}$. This set is essentially a thin rectangle hugging the paraboloid near $\left(N, 0,-N^{2}\right)$ lying above a unit square centered at $(N, 0)$. Note that $\left|R_{N}\right| \sim 1$. Choose $\widehat{\bar{u}}=\chi_{R_{N}}, \widehat{\bar{v}}=\chi_{R_{-N}}$. Geometric considerations imply $\widehat{(\overline{u v})} \sim \frac{1}{N} \chi_{T_{3}}$ where $T_{3}=\left\{(k, \lambda):|k| \lesssim 1,\left|\lambda-\frac{1}{2} N^{2}\right| \lesssim N\right\}$. Indeed, $T_{3} \sim R_{N}+R_{-N}$ and a translate of $R_{N}$ overlaps $R_{-N}$ in a set of size at most $\left|\frac{1}{N} \times 1 \times 1\right| \sim \frac{1}{N}$. Note that $\left|T_{3}\right| \sim$ $|1 \times 1 \times N|=N$. We can now calculate $\|\overline{u v}\|_{X_{\sigma, b-1}} \sim \frac{1}{N}\left(N^{2}\right)^{b-1} N^{\frac{1}{2}} \sim N^{2(b-1)-\frac{1}{2}}$. Also, $\|u\|_{X_{s, b}}=\|v\|_{X_{s, b}} \sim N^{s}$. Therefore, the estimate (2.2) requires that

$$
-(1-b)-\frac{1}{4}<s
$$

Since $b=\frac{1}{2}+$, we require $s>-\frac{3}{4}$.

Now, consider the situation when $\widehat{\bar{u}}=\widehat{\bar{v}}=\chi_{R_{N}}$. We observe that $\widehat{\overline{u v}} \sim \chi_{S_{3}}$ where $S_{3}$ is the translate of (the double of) $R_{N}$ centered at $\left(2 N, 0,2 N^{2}\right)$. Therefore, we can calculate $\|\overline{u v}\|_{X_{\sigma, b-1}} \sim N^{\sigma+2(b-1)}$ and $\|u\|_{X_{s, b}}=\|v\|_{X_{s, b}} \sim N^{s}$. We find that (2.2) requires $\sigma+2(b-1)<2 s$.

The same analysis shows the necessity of the conditions $-\frac{1}{4}-(1-b)<s$ and $\sigma<2 s+2(1-b)$ for (2.3) to hold. Indeed, first take $\widehat{u}=\chi_{R_{N}}, \widehat{v}=\chi_{R_{-N}}$ and then consider $\widehat{u}=\widehat{v}=\chi_{R_{N}}$.

Consider the situation in (2.4) when $\widehat{\bar{u}}=\chi_{\Gamma_{N}}$ and $\widehat{v}=\chi_{R_{N}}$ where $\Gamma_{N}=\{(k, \lambda)$ : $\left.|k+(N, 0)| \leq 1,\left.|\lambda-| k\right|^{2} \mid \leq 1\right\}$. Note that $\Gamma_{N}$ and $R_{N}$ are essentially translates of each other. Simple calculations lead to $\|\bar{u} v\|_{X_{\sigma, b-1}} \sim N^{b-1}$ and to the requirement

$$
-\frac{1}{4}+=\frac{1}{2}(b-1)<s
$$

for 2.4 to hold.

Finally, introduce the sets $P_{0}=\left\{\left(k^{1}, k^{2}, \lambda\right):\left|k^{1}\right|<\frac{1}{N},\left|k^{2}\right|<1,\left.|\lambda \pm| k\right|^{2} \mid \leq\right.$ $1\}, S_{N}=\left\{\left(k^{1}, k^{2}, \lambda\right):\left|k^{1}-N\right|<\frac{1}{N},\left|k^{2}\right|<1,\left.|\lambda+| k\right|^{2} \mid \leq 1\right\}$. (Recall that $k^{1}$ denotes the first component of the 2-vector $k$, etc.) Define $\widehat{\bar{u}}=\chi_{P_{0}}$ and $\widehat{v}=\chi_{S_{N}}$. A calculation reveals that $\widehat{(\bar{u} v)} \sim \frac{1}{N} \chi_{S_{N}}$. So, we find that $\|\bar{u} v\|_{X_{\sigma, b-1}} \sim N^{\sigma-\frac{3}{2}}$. Since $\|v\|_{X_{s, b}} \sim N^{-\frac{1}{2}}$ and $\|u\|_{X_{s, b}} \sim N^{s-\frac{1}{2}}$, the estimate (2.4) requires that $\sigma-\frac{1}{2}<s$. Also, since $P_{0}$ is symmetric under reflection in the $\{\lambda=0\}$ plane, the same condition must hold with $\bar{u}$ replaced by $u$. Hence, (2.3) also requires $\sigma<s+\frac{1}{2}$. A simple modification of this example shows the necessity of the condition $\sigma<s+\frac{1}{2}+2(1-b)$ for (2.2) to hold.

A Multilinear Estimate. We conclude this section with a multilinear estimate which will be combined with (2.2) in the next section to prove regularity bounds on finite energy solutions of certain nonlinear Schrödinger equations. 
Proposition 1. Let $\widetilde{u}$ denote $u$ or $\bar{u}$. Fix a natural number $r$. The multilinear estimate

$$
\left\|\widetilde{u_{1}} \ldots \widetilde{u_{r}}\right\|_{X_{\frac{1}{2}+, \frac{1}{2}+}} \leq \prod_{j=1}^{r}\left\|u_{j}\right\|_{X_{1+, \frac{1}{2}+}}
$$

holds.

The proof does not distinguish between the factors $u$ and $\bar{u}$.

Proof. The case $r=2$ is established in Theorem 2.3 in [13] (Note that the structure $u^{2}$ is not actually used in the proof.). The extra factors in the $r>2$ case are absorbed (essentially) using the fact that $X_{1+, \frac{1}{2}+}$ embeds in $L_{x, t}^{\infty}$.

By duality and the definition of $X_{s, b}$, it suffices to show

$$
\begin{aligned}
& \int_{\substack{\xi=\xi_{1}+\cdots+\xi_{r} \\
\lambda=\lambda_{1}+\cdots+\lambda_{r}}}(1+|\xi|)^{\frac{1}{2}+}\left(1+\left.|\lambda+| \xi\right|^{2} \mid\right)^{\frac{1}{2}+} d(\xi, \lambda) \prod_{j=1}^{r} \frac{\left(1+\left|\xi_{j}\right|\right)^{-1-\epsilon} c_{j}\left(\xi_{j}, \lambda_{j}\right)}{\left(1+\left.\left|\lambda_{j} \pm\right| \xi_{j}\right|^{2} \mid\right)^{\frac{1}{2}+}} \\
& \leq C\|d\|_{L^{2}} \prod_{j=1}^{r}\left\|c_{j}\right\|_{L^{2}},
\end{aligned}
$$

where the \pm choices are arbitrary. The constraint $\lambda=\lambda_{1}+\cdots+\lambda_{r}$ and the triangle inequality imply

$$
\left.|\lambda+| \xi\right|^{2} \mid \lesssim \max \left(\left.\left|\lambda_{1} \pm\right| \xi_{1}\right|^{2}|, \ldots,| \lambda_{r} \pm\left.\left|\xi_{r}\right|^{2}|,| \xi_{1}\right|^{2}, \ldots,\left|\xi_{r}\right|^{2},|\xi|^{2}\right) .
$$

Since $\xi=\xi_{1}+\cdots+\xi_{r}, \max \left(\left|\xi_{1}\right|^{2}, \ldots,\left|\xi_{r}\right|^{2},|\xi|^{2}\right) \lesssim\left|\xi^{*}\right|^{2}$ where $\left|\xi^{*}\right|=\max _{j=1, \ldots, r}\left(\left|\xi_{j}\right|\right)$.

Case 1. $\left.\left|\lambda_{1} \pm\right| \xi_{1}\right|^{2} \mid=\max$ in $(2.33)$.

The left side of (2.32) is estimated

$$
\int_{\substack{\xi=\xi_{1}+\cdots+\xi_{r} \\ \lambda=\lambda_{1}+\cdots+\lambda_{r}}}(1+|\xi|)^{\frac{1}{2}+} d(\xi, \lambda\rangle\left(1+\left|\xi_{1}\right|\right)^{-1-\epsilon} c_{1}\left(\xi_{1}, \lambda_{1}\right) \prod_{j=2}^{r} \frac{\left(1+\left|\xi_{j}\right|\right)^{-1-\epsilon} c_{j}\left(\xi_{j}, \lambda_{j}\right)}{\left(1+\left.\left|\lambda_{j} \pm\right| \xi_{j}\right|^{2} \mid\right)^{\frac{1}{2}+}}
$$

Case 1A. $\left|\xi_{1}\right|=\left|\xi^{*}\right|$.

We have $(1+|\xi|)^{\frac{1}{2}+}\left(1+\left|\xi_{1}\right|\right)^{-1-\epsilon} \leq 1$ and estimate in $L_{x, t}^{2} L_{x, t}^{2} \prod_{j=2}^{r} L_{x, t}^{\infty}$ using a standard argument involving Fourier transform properties, Hölder's inequality and then the Sobolev embedding theorem.

Case 1B. $\left|\xi_{k}\right|=\left|\xi^{*}\right|$ for some $k \in\{2, \ldots, r\}$. 
Without loss, we may take $k=2$ by renaming the variables. In this situation, we estimate

$$
\begin{gathered}
\int_{\substack{\xi=\xi_{1}+\cdots+\xi_{r} \\
\lambda=\lambda_{1}+\cdots+\lambda_{r}}} d(\xi, \lambda)\left(1+\left|\xi_{1}\right|\right)^{-1-\epsilon} c_{1}\left(\xi_{1}, \lambda_{1}\right) \frac{\left(1+\left|\xi_{2}\right|\right)^{-\frac{1}{2}-\frac{1}{2} \epsilon} c_{2}\left(\xi_{2}, \lambda_{2}\right)}{\left(1+\left.\left|\lambda_{2} \pm\right| \xi_{2}\right|^{2} \mid\right)^{\frac{1}{2}+}} \\
\times \prod_{j=3}^{r} \frac{\left(1+\left|\xi_{j}\right|\right)^{-1-\epsilon} c_{j}\left(\xi_{j}, \lambda_{j}\right)}{\left(1+\left.\left|\lambda_{j} \pm\right| \xi_{j}\right|^{2} \mid\right)^{\frac{1}{2}+}} .
\end{gathered}
$$

This may be done in $L_{t}^{2} L_{x}^{2} L_{t}^{2} L_{x}^{\infty} L_{t}^{\infty} L_{x}^{2} \prod_{j=3}^{r} L_{t}^{\infty} L_{x}^{\infty}$. The cases when $\left.\left|\lambda_{j} \pm\right| \xi_{j}\right|^{2} \mid=$ $\max$ in (2.33) for $2 \leq j \leq r$ are similar.

Case 2. $\left|\xi^{*}\right|^{2}=\max$ in (2.33).

We may suppose $\left|\xi_{1}\right|=\left|\xi^{*}\right|$. We need to bound

$$
\int_{\substack{\xi=\xi_{1}+\cdots+\xi_{r} \\ \lambda=\lambda_{1}+\cdots+\lambda_{r}}}(1+|\xi|)^{\frac{1}{2}} d(\xi, \lambda) \frac{c_{1}\left(\xi_{1}, \lambda_{1}\right)}{\left(1+\left.\left|\lambda_{1} \pm\right| \xi_{1}\right|^{2}\right)^{\frac{1}{2}+}} \prod_{j=2}^{r} \frac{\left(1+\left|\xi_{j}\right|\right)^{-1-\epsilon} c_{j}\left(\xi_{j}, \lambda_{j}\right)}{\left(1+\left.\left|\lambda_{j} \pm\right| \xi_{j}\right|^{2} \mid\right)^{\frac{1}{2}+}} .
$$

Since $|\xi| \lesssim\left|\xi^{*}\right|$, we may write

$$
\begin{aligned}
& \int_{\substack{\xi=\xi_{1}+\cdots+\xi_{r} \\
\lambda=\lambda_{1}+\cdots+\lambda_{r}}} d(\xi, \lambda)\left(\frac{\left(1+\left|\xi_{1}\right|\right)^{\frac{1}{2}} c_{1}\left(\xi_{1}, \lambda_{1}\right)}{\left(1+\left.\left|\lambda_{1} \pm\right| \xi_{1}\right|^{2}\right)^{\frac{1}{2}+}} \frac{\left(1+\left|\xi_{2}\right|\right)^{-1-\epsilon} c_{2}\left(\xi_{2}, \lambda_{2}\right)}{\left(1+\left.\left|\lambda_{2} \pm\right| \xi_{2}\right|^{2}\right)^{\frac{1}{2}+}}\right) \\
& \quad \times \prod_{j=3}^{r} \frac{\left(1+\left|\xi_{j}\right|\right)^{-1-\epsilon} c_{j}\left(\xi_{j}, \lambda_{j}\right)}{\left(1+\left.\left|\lambda_{j} \pm\right| \xi_{j}\right|^{2} \mid\right)^{\frac{1}{2}+}} .
\end{aligned}
$$

We estimate this expression via Hölder's inequality in $L_{x, t}^{2} L_{x, t}^{2} \prod_{j=3}^{r} L_{x, t}^{\infty}$. Next, we apply the Sobolev inequality to get

$$
\|d\|_{L^{2}}\|F G\|_{L^{2}} \prod_{j=3}^{r}\left\|c_{j}\right\|_{L^{2}}
$$

where

$$
\widehat{F}(\xi, \lambda)=\frac{\left(1+\left|\xi_{1}\right|\right)^{\frac{1}{2}} c_{1}\left(\xi_{1}, \lambda_{1}\right)}{\left(1+\left.\left|\lambda_{1} \pm\right| \xi_{1}\right|^{2}\right)^{\frac{1}{2}+}}
$$

and

$$
\widehat{G}(k, \lambda)=\frac{\left(1+\left|\xi_{2}\right|\right)^{-1-\epsilon} c_{2}\left(\xi_{2}, \lambda_{2}\right)}{\left(1+\left.\left|\lambda_{2} \pm\right| \xi_{2}\right|^{2}\right)^{\frac{1}{2}+}} .
$$

Note that Cauchy-Schwarz and the $L^{4}$ Strichartz estimate (2.24) shows that $\|F G\|_{L_{x t}^{2}} \leq\|F\|_{L_{x t}^{4}}\|G\|_{L_{x t}^{4}} \leq C\|F\|_{X_{0, b}}\|G\|_{X_{0, b}} \leq C\left\|c_{1}\right\|_{L_{t}^{2} H_{x}^{\frac{1}{2}}}\left\|c_{2}\right\|_{L_{t}^{2} H_{x}^{-1-\epsilon}}$ which 
is not good enough. We transport $\frac{1}{2}$ derivative from $F$ to $G$ using Bourgain's Strichartz refinement [3] to obtain

$$
\|F G\|_{L^{2}} \leq\left\|c_{1}\right\|_{L^{2}}\left\|c_{2}\right\|_{H^{-\frac{1}{2}-\epsilon}} \leq\left\|c_{1}\right\|_{L^{2}}\left\|c_{2}\right\|_{L^{2}}
$$

and Case 2 follows.

\section{REgularity of GLOBAL-IN-TIME AND BLOW-UP SOLUTIONS}

How does the spatial regularity of solutions of the initial value problem

$$
\left\{\begin{array}{c}
i u_{t}+\Delta u \pm|u|^{2} u=0 \\
u(0)=\phi \in \mathcal{S}\left(\mathbb{R}^{2}\right)
\end{array}\right.
$$

evolve? Conservation of the Hamiltonian

$$
H(u, \bar{u})=\int_{\mathbb{R}^{2}} \frac{1}{2}|\nabla u|^{2} \mp \frac{1}{4}|u|^{4} d x
$$

and the $L^{2}$ norm $\|u(t)\|_{L^{2}}=\|\phi\|_{L^{2}}$ implies a priori $H^{1}$ control $\left(\|u(t)\|_{H^{1}} \leq\right.$ $\left.C\|\phi\|_{H^{1}}\right)$ for all time in the defocusing case ( - in (3.1); + in $(3.2)$ ) and also in the focusing case for small enough $\|\phi\|_{L^{2}}$. In the presence of a priori $H^{1}$ control, the available local wellposedness theory [5] iterates to imply global wellposedness. Absent $H^{1}$ control, solutions of the focusing (3.1) may satisfy

$$
\left\|\nabla_{x} u(t)\right\|_{L^{2}} \rightarrow \infty \text { as } t \rightarrow T^{*}<\infty .
$$

For global-in-time solutions satisfying a priori $H^{1}$ control, how does $\|u(t)\|_{H^{s}}$, $s \gg 1$ behave as $t \rightarrow \infty$ ? Bourgain showed [2] that a refined local-in-time analysis implied $\|u(t)\|_{H^{s}} \leq C|t|^{2(s-1)+}$ which provides a substantial improvement over the easy exponential bound. Staffilani proved in [12], [13] that $\|u(t)\|_{H^{s}} \leq C|t|^{(s-1)+}$ by a direct argument exploiting an estimate on the bilinearity $\overline{u v}$ in the scale of $X_{s, b}$ spaces. Insertion of the sharp bilinear estimate (2.2) into Staffilani's argument gives the following result.

Theorem 2. Consider initial data $\phi$ satisfying conditions implying that the associated solution of (3.1) satisfies $\|u(t)\|_{H^{1}} \leq C\|\phi\|_{H^{1}}$. Then, for $s \gg 1$,

$$
\|u(t)\|_{H^{s}} \leq C|t|^{\alpha(s)} \text { as }|t| \rightarrow \infty
$$

where

$$
\alpha(s)=\frac{2}{3}(s-1)+.
$$

For blow-up solutions (3.3) of (3.1), how does $\|u(t)\|_{H^{s}}, s \gg 1$, behave as $t \rightarrow T^{*}$ ? Cazeneve and Weissler [5] proved that for $t$ near $T^{*}$,

$$
\left\|\nabla_{x} u(t)\right\|_{L^{2}} \gtrsim\left(T^{*}-t\right)^{-\frac{1}{2}}
$$

The explicit blow-up solutions obtained using conformal invariance of (3.1) satisfy

$$
\left\|\nabla_{x} u(t)\right\|_{L^{2}} \sim\left(T^{*}-t\right)^{-1} .
$$

It is conjectured, based in part on numerical evidence [10], that $C\left(T^{*}-t\right)^{-1}$ is an upper bound on the blow-up rate of $\left\|\nabla_{x} u(t)\right\|_{L^{2}}$. This appears to be a hard problem. We thank Frank Merle for suggesting there may be a connection between the global-in-time and blow-up regularity bounds. Theorem 2 above has the following implication. 
Corollary 3.1. Assume that $u(t)$ is a blow-up solution of (3.1) with blow-up occurring at time $T^{*}, 0<T^{*}<\infty$. Assume furthermore that for $t \in\left[0, T^{*}[\right.$

$$
\sup _{\tau \in[0, t]}\|\nabla u(\tau)\|_{L_{x}^{2}}=\lambda(t) \text {. }
$$

Then, for $s \gg 1$,

$$
C^{\prime}[\lambda(t)]^{s} \leq\|u(t)\|_{H_{x}^{s}} \leq C[\lambda(t)]^{2 \alpha(s)+s}
$$

where $\alpha(s)$ is the degree of the polynomial upperbound on the $H^{s}$ norm of globalin-time solutions satisfying a priori $H^{1}$ control.

Proof. Fix $t$ near $T^{*}$. Then $\lambda(t)=\lambda$ is defined. Introduce the rescaling

$$
v(x, \tau)=\frac{1}{\lambda} v\left(\frac{x}{\lambda}, \frac{\tau}{\lambda^{2}}\right) .
$$

The function $v$ solves

$$
\left\{\begin{array}{c}
i \partial_{\tau} v+\Delta v+|v|^{2} v=0, \tau \in\left[0, \lambda^{2} t\right] \\
v(0)=\frac{1}{\lambda} u\left(\frac{x}{\lambda}, 0\right)
\end{array}\right.
$$

and

$$
\|\nabla v(\tau)\|_{L_{x}^{2}} \leq 1, \quad \tau \in\left[0, \lambda^{2} t\right] .
$$

Notice that $\lambda^{2} t$ is a huge number so the function $v$ is defined for a very long interval of $\tau$ and that (3.12) together with $L_{x}^{2}$ gives an a priori bound on $\|v(\tau)\|_{H^{1}}$. Theorem 2 gives

$$
\left\|\nabla^{s} v(\tau)\right\|_{L_{x}^{2}} \lesssim\left[\lambda^{2} t\right]^{\alpha(s)}, \quad \tau \in\left[0, \lambda^{2} t\right]
$$

as a consequence of certain multilinear estimates as will be shown in the proof below. The definition of $v$ in terms of $u$ shows that

$$
\lambda^{-s}\left\|\nabla^{s} u\left(\cdot, \frac{\tau}{\lambda^{2}}\right)\right\|_{L_{x}^{2}}=\left\|\nabla^{s} v(\cdot, \tau)\right\|_{L_{x}^{2}} .
$$

Finally, note that $t$ appearing on the right side of 3.13 is bounded by the constant $T^{*}$. Combining (3.14) and (3.13) gives the upper estimate in the corollary. The lower estimate follows by interpolating the $H^{1}$ norm between the $L^{2}$ and $H^{s}$ norms.

We now revisit the argument of Staffilani [12] and prove Theorem 2,

Proof. Let $s=2 n, n \in \mathbb{N}$ for convenience. We are considering (3.1) with initial data satisfying $H^{1}$ control. Our goal is to bound $\|u(t)\|_{H^{s}}$ from above as $t \rightarrow \infty$. Let $T$ be the lifetime of the local wellposedness result which is bounded from below by a negative power of $\|\phi\|_{H^{1}}$. By a priori $H^{1}$ control, the local result iterates to prove global wellposedness.

Following Bourgain [2], we wish to show the local-in-time estimate

$$
\sup _{t \in[0, T]}\|u(t)\|_{H^{s}} \leq\|u(0)\|_{H^{s}}+\|u(0)\|_{H^{s}}^{1-\delta}
$$

which iterates to give $\|u(t)\|_{H^{s}} \leq C|t|^{\frac{1}{\delta}}$. 
By $L^{2}$ conservation and interpolation, it suffices to control the homogeneous norm $\|u(t)\|_{\dot{H}^{s}}$. The fundamental theorem of calculus gives

$$
\begin{aligned}
\|u(t)\|_{\dot{H}^{s}}^{2}-\|u(0)\|_{\dot{H}^{s}}^{2} & =\int_{0}^{t} \frac{d}{d \tau}\|u(\tau)\|_{\dot{H}^{s}}^{2} \\
& =2 \Re \int_{0}^{t}\left\langle B^{s} \dot{u}(\tau), B^{s} u(\tau)\right\rangle d \tau
\end{aligned}
$$

where $\langle f, g\rangle=\int f \bar{g} d x$ is the standard $L_{x}^{2}$ inner product and $B=\sqrt{-\Delta}$. Using the equation we obtain

$$
=2 \Re i \int_{0}^{t}\left\langle B^{s}(-\Delta u), B^{s} u\right\rangle d \tau \pm 2 \Re i \int_{0}^{t}\left\langle B^{s}\left(|u|^{2} u\right), B^{s} u\right\rangle d \tau .
$$

The first integral is real so it disappears. The worst terms arise when all of $B^{s}$ hits $u$ or all of $B^{s}$ hits $\bar{u}$. However, if all of $B^{s}$ hits $u$, the resulting integrand is real and so it disappears. Therefore, we can write,

$$
\|u(t)\|_{\dot{H}^{s}}^{2}-\|u(0)\|_{\dot{H}^{s}}^{2} \leq C\left|\int_{0}^{t} u^{2}\left(B^{s} \bar{u}\right)^{2} d x d t\right|+\text { l.o.t. },
$$

where l.o.t. stands for lower order terms in which the differentiation is shared among the factors. Plancherel followed by Cauchy-Schwarz (with weights) shows the integral is estimated by

$$
\left\|\bar{u}^{2}\right\|_{X_{\frac{1}{2}, \frac{1}{2}}}\left\|\left(B^{s} \bar{u}\right)^{2}\right\|_{X_{-\frac{1}{2},-\frac{1}{2}}} .
$$

Theorem 1 establishes (up to a loss of $\epsilon$ ) the bilinear estimate

$$
\|\overline{u v}\|_{X_{-\frac{1}{2},-\frac{1}{2}}^{T}} \leq C\|u\|_{X_{-\frac{3}{4}, \frac{1}{2}}}\|v\|_{X_{-\frac{3}{4}, \frac{1}{2}}^{T}} .
$$

The other term is estimated using Proposition 1 giving (again up to $\epsilon$ )

$$
\left\|\bar{u}^{2}\right\|_{X_{\frac{1}{2}, \frac{1}{2}}^{T}} \leq C\left(\|u\|_{X_{1, \frac{1}{2}}^{T}}\right)^{2} .
$$

The local wellposedness result of Bourgain [1] implies, for $\mu>0$, that

$$
\|u\|_{X_{\mu, b}^{T}} \leq C\|u(0)\|_{H^{\mu}} .
$$

Therefore, applying (3.18), (3.19), (3.20) and exploiting $H^{1}$ control, we obtain

$$
\|u(t)\|_{\dot{H}^{s}}^{2}-\|u(0)\|_{\dot{H}^{s}}^{2} \leq C\|u(0)\|_{H^{s-\frac{3}{4}}}^{2} .
$$

Upon interpolating $\|u(0)\|_{H^{s-\frac{3}{4}}}$ between $\|u(0)\|_{H^{s}}$ and $\|u(0)\|_{H^{1}}$, we obtain (3.15) for the top order term. A similar argument applies to the lower order terms.

Comments on Higher Power Semilinear NLS Equations on $\mathbb{R}^{2}$. Consider a global-in-time solution $u$ of the defocusing NLS equation

$$
\left\{\begin{array}{c}
i u_{t}+\Delta u-|u|^{p-1} u=0, \quad u: \mathbb{R}_{x}^{2} \times \mathbb{R}_{t} \longmapsto \mathbb{C}, \\
u(0)=\phi,
\end{array}\right.
$$

with finite energy

$$
E=E(u)=\int_{\mathbb{R}^{2}}|\nabla u|^{2}+|u|^{2}+\frac{2}{p+1}|u|^{p+1} d x .
$$


Nakanishi recently proved [1] for $p>3$ that such solutions satisfy

$$
\|u\|_{L^{2(p-1)}\left(\mathbb{R}_{x}^{2} \times \mathbb{R}_{t}\right)} \leq C(E) .
$$

In case $p-1$ is even, we infer from this inequality that smooth global-in-time finite energy solutions of (3.22) satisfy

$$
\|u(t)\|_{H^{s}} \leq C
$$

We restrict our attention to $p-1$ even, so that $u \longmapsto|u|^{p-1} u$ is smooth. We imitate an argument appearing in 4 .

1. A calculation shows, for $s \geq 1, I=[0, b], 0<b \leq \infty$,

$$
\left\|D_{x}^{s} u\right\|_{L_{x, t \in I}^{4}} \leq c_{1}\|\phi\|_{H^{s}}+c_{2}\|u\|_{L_{x, t \in I}^{2(p-1)}}^{p-1}\left\|D_{x}^{s} u\right\|_{L_{x, t \in I}^{4}} .
$$

Indeed, from the integral equation, we have

$$
\left\|D_{x}^{s} u\right\|_{L_{x, t \in I}^{4}} \leq\left\|D_{x}^{s} e^{i t \Delta} \phi\right\|_{L_{x, t \in I}^{4}}+\left\|D_{x}^{s} \int_{0}^{t} e^{i(t-\tau) \Delta}|u|^{p-1} u d \tau\right\|_{L_{x, t \in I}^{4}} .
$$

Strichartz inequality in $\mathbb{R}_{x}^{2} \times \mathbb{R}_{t}$ gives

$$
\left\|D_{x}^{s} e^{i t \Delta} \phi\right\|_{L_{x, t \in I}^{4}} \leq c_{1}\|\phi\|_{H^{s}}
$$

and the inhomogeneous version implies

$$
\left\|D_{x}^{s} \int_{0}^{t} e^{i(t-\tau) \Delta} v d \tau\right\|_{L_{x, t \in I}^{4}} \leq C\left\|D_{x}^{s} v\right\|_{L_{x, t \in I}^{\frac{4}{3}}} .
$$

We write

$$
\left\|D_{x}^{s}|u|^{p-1} u\right\|_{L_{x, t \in I}^{\frac{4}{3}}} \leq C\left\|\left(D_{x}^{s} u\right)|u|^{p-1}\right\|_{L_{x, t \in I}^{\frac{4}{3}}}+\text { l.o.t. }
$$

where l.o.t. denotes lower order terms where the differentiation is shared among the factors. By Hölder, we have

$$
\left\|\left(D_{x}^{s} u\right)|u|^{p-1}\right\|_{L_{x, t \in I}^{\frac{4}{3}}} \leq\left\|D_{x}^{s} u\right\|_{L_{x, t \in I}^{4}}\|u\|_{L_{x, t \in I}^{2(p-1)}}^{p-1}
$$

as claimed. The lower order terms can be handled with the Leibniz rule and interpolation using the smoothness of the nonlinearity.

2. A similar calculation shows for $s \geq 1$ that

$$
\sup _{t \in I}\|u(t)\|_{H^{s}} \leq c_{3}\|\phi\|_{H^{s}}+c_{4}\|u\|_{L_{x, t \in I}^{2(p-1)}}^{p-1}\left\|D_{x}^{s} u\right\|_{L_{x, t \in I}^{4}} .
$$

3. Fix $\epsilon>0$, by (3.24) there exist disjoint intervals $I_{1}, \ldots, I_{J}, J=J(\epsilon)<\infty$, such that

$$
R_{t}=\bigcup_{j=1}^{J} I_{j}
$$

and, by (3.24),

$$
\|u\|_{L_{x, t \in I}^{2(p-1)}}<\epsilon
$$

uniformly in $j$. Therefore, for small enough $\epsilon$, we can absorb the nonlinear terms in (3.26) and (3.27) into the left side of the inequalities. Finally, we note that there are finitely many $I_{j}$ so (3.27) implies (3.25). 
Remark 4. If we consider the evolution $i \partial_{t} u+\Delta u+|u|^{p-1} u=0, p>3$, in place of the evolution in (3.1), the Cauchy-Schwarz application at (3.17) leads to

$$
\left\|\bar{u}^{\frac{p+1}{2}} u^{\frac{p-3}{2}}\right\|_{X_{\frac{1}{2}, \frac{1}{2}}}\left\|\left(B^{s} \bar{u}\right)^{2}\right\|_{X_{-\frac{1}{2},-\frac{1}{2}}} .
$$

Proposition 1 allows us to estimate the first factor. An adaptation of the proof of Theorem 2, exploiting the $r>2$ case of Proposition 1 (see Remark 4), shows all such solutions satisfy (3.4) provided $p-1$ is even. It is not known if the smooth global-in-time finite energy solutions of this focusing analog of (3.22) satisfy the estimate (3.25).

\section{REFERENCES}

1. J. Bourgain, Fourier transform restriction phenomena for certain lattice subsets and applications to nonlinear evolution equations. I,II, Geom. Funct. Anal. 3 (1993), 107-156, 209-262. MR 95d:35160a MR 95d:35160b

2. - On the growth in time of higher Sobolev norms of smooth solutions of Hamiltonian PDE, Internat. Math. Res. Notices 6 (1996), 277-304. MR 97k:35016

3. - Refinements of Strichartz' inequality and applications to $2 D$-NLS with critical nonlinearity, International Mathematical Research Notices 5 (1998), 253-283. MR 99f:35184

4. Global solutions of nonlinear Schrödinger equations, American Mathematical Society, Colloquium Publications, 46, Providence, RI, 1999. MR 2000h:35147

5. T. Cazenave and F. B. Weissler, The Cauchy problem for the critical nonlinear Schrödinger equation in $H^{s}$, Nonlinear Anal. 14 (1990), 807-836. MR 91j:35252

6. J. E. Colliander, C. E. Kenig, and G. Staffilani, An $X_{s, b}$ Space Approach to Local Wellposedness of the KP-I Equation, in preparation.

7. J.-M. Delort and D. Fang, Almost global existence for solutions of semilinear Klein-Gordon equations with small weakly decaying Cauchy data 25 (2000), no. 11-12, 2119-2169.

8. C. Kenig, G. Ponce, and L. Vega, A bilinear estimate with applications to the KdV equation, J. Amer. Math. Soc. 9 (1996), 573-603. MR 96k:35159

9. - Quadratic forms for the 1-D semilinear Schrödinger equation., Trans. Amer. Math. Soc. 348 (1996), 3323-3353. MR 96j:35233

10. B. LeMesurier, G. Papanicolaou, C. Sulem, and P.-L. Sulem, The focusing singularity of the nonlinear Schrödinger equation, Directions in partial differential equations (Madison, WI, 1985), Academic Press, Boston, MA, 1987, pp. 159-201. MR 90i:35249

11. K. Nakanishi, Energy scattering for nonlinear Klein-Gordon and Schrödinger equations in spatial dimensions 1 and 2, J. Funct. Anal. 169 (1999), 201-225. MR 2000m:35141]

12. G. Staffilani, On the growth of high Sobolev norms of solutions for KdV and Schrödinger equations, Duke Math. J. 86 (1997), 109-142. MR 98b:35192

13. 79-107. MR 98b:35191

14. T. Tao, Multilinear weighted convolution of $L^{2}$ functions and applications to nonlinear dispersive equations, to appear, Amer. J. Math.

Department of Mathematics, University of California, Berkeley, California 94720

E-mail address: colliand@math. berkeley.edu

Département of Mathématiques, Université de Paris-Nord, 93430 Villetaneuse, FRANCE

E-mail address: delort@math.univ-paris13.fr

Department of Mathematics, University of Chicago, Chicago, Illinois 60637

E-mail address: cek@math.uchicago.edu

Department of Mathematics, Stanford University, Stanford California 94305

E-mail address: gigliola@math.stanford.edu 